\title{
Stiffness Analysis and Experimental Verification of Segment Frame of Continuous Casting Machine
}

\author{
Zhufeng Lei ${ }^{1, a}$, Wenbin Su ${ }^{1}$, Bingrui Hou ${ }^{1}$ and Qi Gao ${ }^{2}$ \\ ${ }^{1}$ Xi'an Jiaotong University, School of Mechanical Engineering, Xi'an, Shaanxi 710049, P.R. China. \\ ${ }^{2}$ China National Heavy Machinery Research Institute Co.,Ltd, Xi'an, Shaanxi 710016, P.R. China.
}

\begin{abstract}
To verify the stiffness, the natural vibration and the reliability of the continuous casting machine in the configuration of dynamic soft reduction. Firstly, the three-dimensional model of the segment frame was established and the finite element simulation of the structure was carried out. Then, the deviation of the roll gap in the working process of the caster was measured by analog loading test. The results show that the stiffness of the segment frame of the continuous casting machine meets the requirements. Using dynamic soft reduction can improve the centerline segregation of casting slab in the process of continuous casting, which is of important practical significance in the production practice.
\end{abstract}

\section{Introduction}

Since the 70 s of the 20th century, with the improvement of the quality requirements of the steel slabs of various countries, the center segregation of the slab has become the critical attention of all countries. Therefore, in order to solve the slab segregation problem, dynamic soft reduction technology is widely used in continuous casting production process [1-3]. At present, SMS Siemag, Siemens VAI and Danieli Davy, which have developed relatively mature dynamic soft reduction equipment and process technology and are successfully applied to the steel industry, Distington). The companies mentioned above have developed a configuration of soft caster fan section equipment and related technologies.

The segregation of high-strength steel series in the CCC pilot production center is prominent due to the inability to meet the requirements of stable and continuous production process quality because the continuous casting machine segments do not have the functions of remote adjustment of roll gap and un-configured dynamic soft reduction [1-2]. In order to minimize centerline segregation and improve the microstructural homogeneity of the strand in subsequent rolling, technologies such as electromagnetic stirring [4-5], thermal soft reduction [6-9], dynamic soft reduction [10-17] and mechanical forging [18] have been applied in the continuous casting of steel. Among all methods, dynamic soft reduction is considered the most flexible and effective way to minimize segregation of cast steel. The dynamic soft reduction technique can be applied to minimize the centerline segregation and porosity by reducing the strand near the final solidification region to compensate for solidification shrinkage [19]. However, there is a higher requirement on the rigidity of caster frame with dynamic soft reduction.

In order to verify whether the rigidity and dynamic characteristics of the caster segment frame meet the requirements and ensure the service life of the equipment after the configuration of dynamic soft reduction. In this paper, the finite element simulation of the structural rigidity and dynamic

\footnotetext{
${ }^{\mathrm{a}}$ Corresponding author: leizhufeng@qq.com
} 
characteristics of the segment frame of a continuous casting machine was carried out. Then, hydraulic jacks were used to simulate the pressure load of the slab casting during continuous casting process to detect the actual segment stiffness of the frame characteristics.

\section{The establishment of segment frame model}

\subsection{The establishment of three-dimensional solid model of segment frame}

The three-dimensional model of the continuous casting machine segment frame was established and the finite element simulation analysis was carried out, as shown in Figure.1.
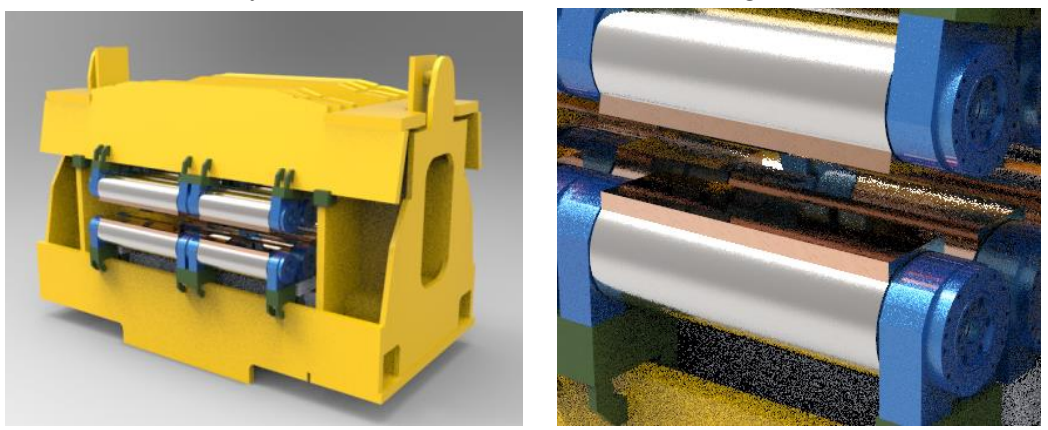

Figure 1. Segment frame three-dimensional model Figure 2. Simulation of slab contact with the nip roll

In order to simulate the reaction force of the slab against the segment frame in the continuous casting process and to simulate the contact between the slab and the segment nip roller, a concave unit was established to minimize the contact between the slab and the nip roll. The simulated slab contact with the nip roll is shown in Figure.2.

\subsection{The establishment of finite element model of segment frame}

When the finite element model of the continuous casting machine fan frame was established, the material selection is Q345B, the density $\rho=7850 \mathrm{~kg} / \mathrm{m}^{3}$, the elastic modulus $\mathrm{E}=2 \mathrm{GPa}$ and the Poisson's ratio $\mu=0.3$. The total mass of the frame is $25364 \mathrm{~kg}$. Due to the meshing process, the hexahedral mesh is more likely to converge than the tetrahedral mesh and the discretization error is small and the precision is high. Therefore, in the design simulation module, the solid model is automatically meshed using a hexahedral mesh to obtain a finite element model, with a total of 977760 nodes and 442188 finite elements, as shown in Figure.3.

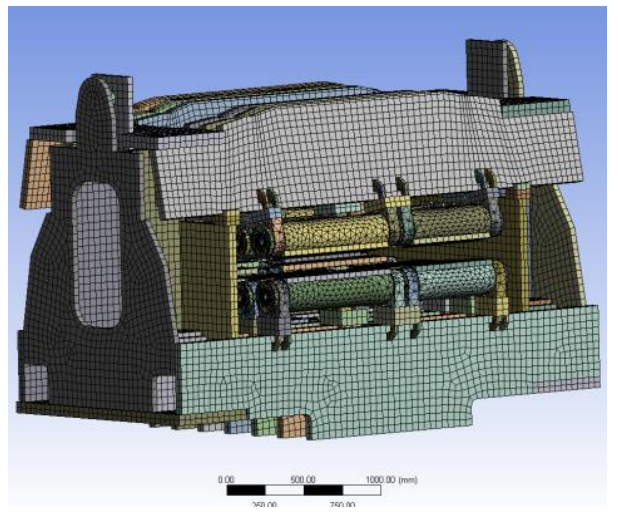

Figure 3. Segment frame finite element model 


\section{Finite element analysis of segment frame}

\subsection{Static analysis of segment frame}

When the segment is working, the sector frame is mainly affected by the reaction force of the slab against the frame, the gravity of the machine frame, the clamping force of the upper and lower clamps, the bulging force, the resistance of the light pressure and the rotation resistance of the roller. Therefore, for the moment, the force loads of the segment frame are:

(1) The weight of the base itself.

(2) The reaction to the segment frame resulting from the compression of the slab.

(3) The clamping force of the upper and lower clamps.

(4) The bulging force.

(5) Resistance of the light pressure and the rotation resistance of the roller.

According to mechanical analysis, during the process of passing through the slab liquid core, the rollers of the inner and outer arc frames are deformed by the pressure, and the deformation of the outer arc frame due to the complete restraint of the outer arc frame is small. In this case, the inner arc frame can be regarded as a simply supported beam model subjected to uniform force, as shown in Figure.4.

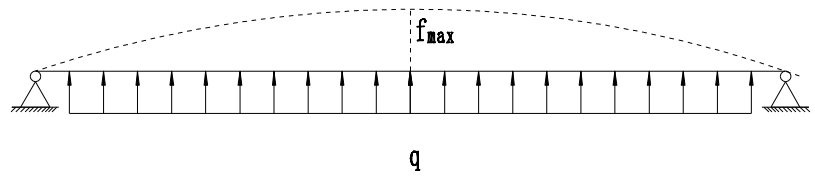

Figure 4. Simply supported beam load deformation diagram

According to mechanical analysis of the mechanics of simply supported beam, under the uniform load, the simply supported beam is deformed [20]. According to the deflection equation:

$$
f_{\max }=\frac{5 q l^{4}}{384 E I}
$$

In which: $f_{\max }$ is the maximum deflection; $q$ is uniform load; $l$ is simply supported beam length; $E$ is modulus of elasticity; $I$ is Sectional moment of inertia.

It can be seen that the maximum deformation occurs at the midpoint of the beam. The maximum deformation of the segment frame occurs at the midpoint of the nip roller connection at both ends of the inner arc frame.

The segment frame is mainly affected by the reaction force of the slab against the frame, assuming that it is evenly distributed along the axial direction of the nip roller. It can be seen from Figure.4 that the force of each row of nip rollers is $300 \mathrm{kN}$ and the deformation of the whole segment frame is less than $1.8 \mathrm{~mm}$. According to equation:

$$
P=\frac{W}{S}=\frac{75000}{0.082^{2} \pi / 4}
$$

The hydraulic test system needs to provide a maximum pressure of $14.8 \mathrm{MPa}$; there are 2 loads applied to the stand for the FEA:

(1) Slab pressure on the frame.

(2) The gravity of the base itself.

According to the analysis results, the equivalent displacement cloud frame of the segment frame is shown in Figure 5. The maximum deformation of the frame appears at the nip roller connecting ends of the inner arc frame with the maximum deformation of $1.8718 \mathrm{~mm}$. The maximum deformation of the segment frame is shown in Figure 6. 


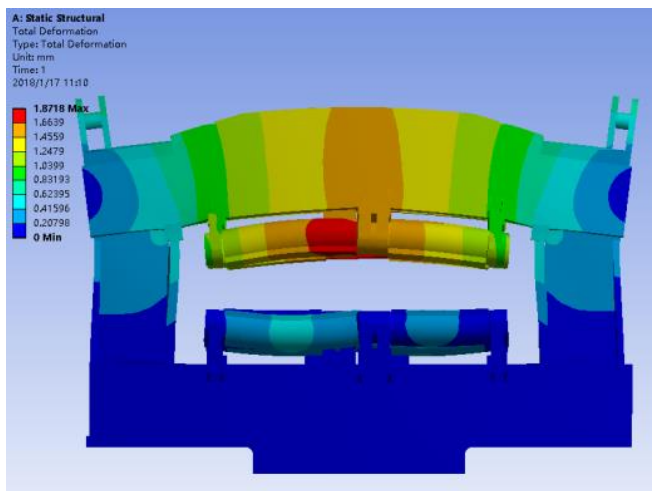

Figure 5. Sector frame equivalent displacement cloud chart

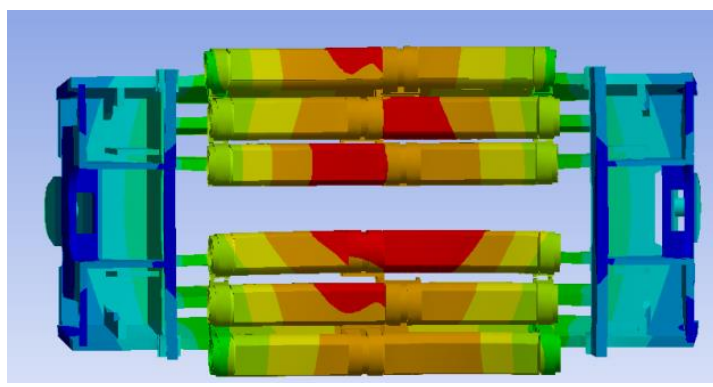

Figure 6. Maximum deformation of the segment frame

According to the theoretical analysis, the deformation of the segment frame occurs mainly in the connecting part of the nip roller of the inner arc frame, and the simulation results are in consistent with the theoretical analysis.

\subsection{Dynamic analysis of segment frame}

During the process of continuous casting, the slab liquid core passes through the segment frame double row nip rollers and is casted under the pressure of driving rollers. During the process of continuous casting, the slab passes through the segment frame while being subjected to great pressure of nip rollers. This process inevitably generates resonance. When the vibration frequency reaches the resonance frequency of the segment frame, the device generates resonance. Therefore, the dynamic analysis of the segment frame provides the basis for the structure anti-vibration and vibration reduction and the improvement of the overall structural reliability and service life.

The boundary conditions of the segment frame are loaded to solve the first six natural frequencies and modes of the segment frame under the pre-stress. The results are shown in Table1, the mode shapes are shown in Figure7 Figure12.

Table 1. The first six natural frequencies and mode shapes analysis of segment frame

\begin{tabular}{|c|c|c|}
\hline & Frequency/Hz & Mode shape \\
\hline $1^{\text {st }}$ order & 47.384 & The swing of inner arc frame swing in the X direction \\
\hline $2^{\text {nd }}$ order & 55.585 & The second-orderly swing of inner arc frame in the X direction \\
\hline $3^{\text {rd }}$ order & 61.628 & The torsion of inner arc frame in the Y direction \\
\hline $4^{\text {th }}$ order & 85.724 & The swing of outer arc frame in the X direction \\
\hline $5^{\text {th }}$ order & 97.263 & The second-orderly swing of outer arc frame in the X direction \\
\hline $6^{\text {th }}$ order & 100.46 & The torsion of inner arc frame in the X direction \\
\hline
\end{tabular}




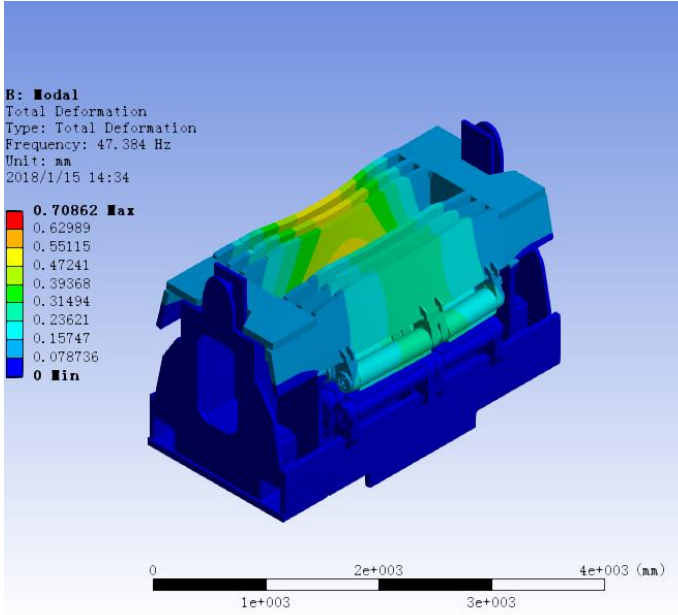

Figure 7. 1st order mode shape cloud chart

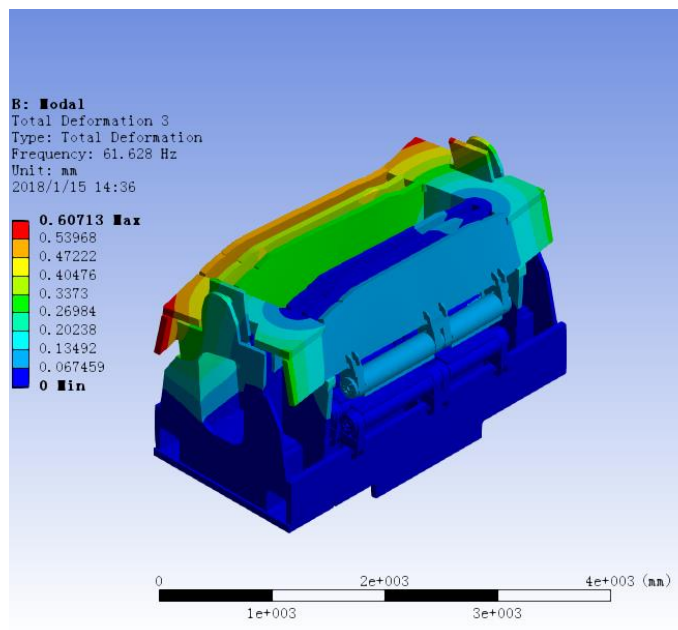

Figure 9. 3rd order mode shape cloud chart

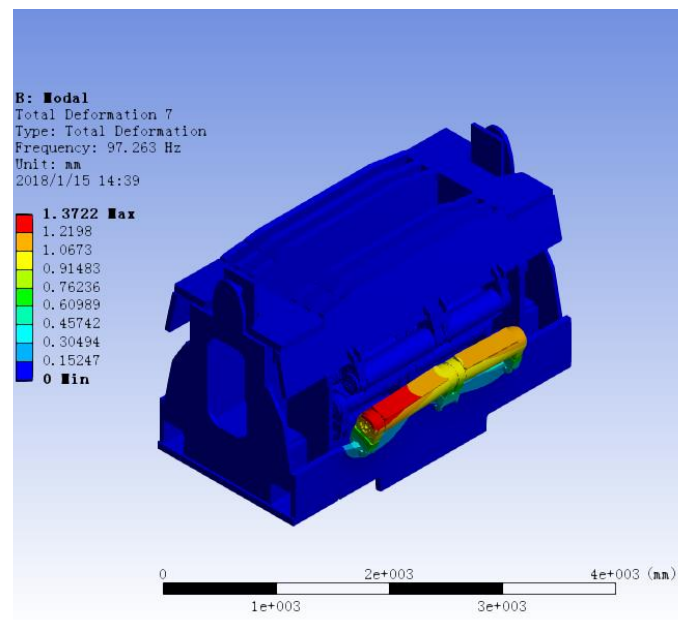

Figure 11. 5th order mode shape cloud chart

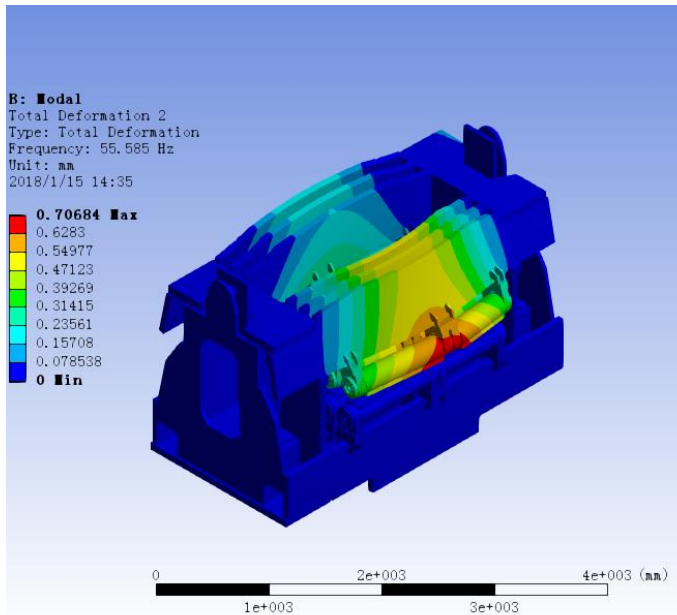

Figure 8. 2nd order mode shape cloud chart

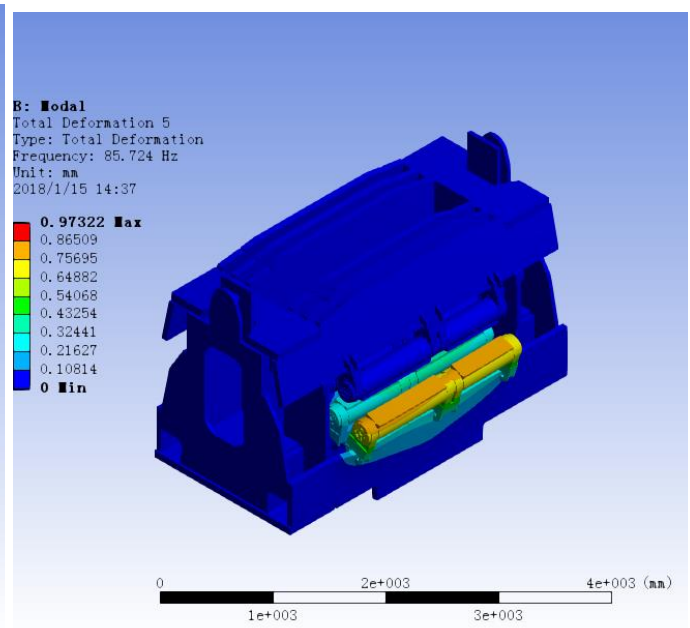

Figure 10. 4th order mode shape cloud chart

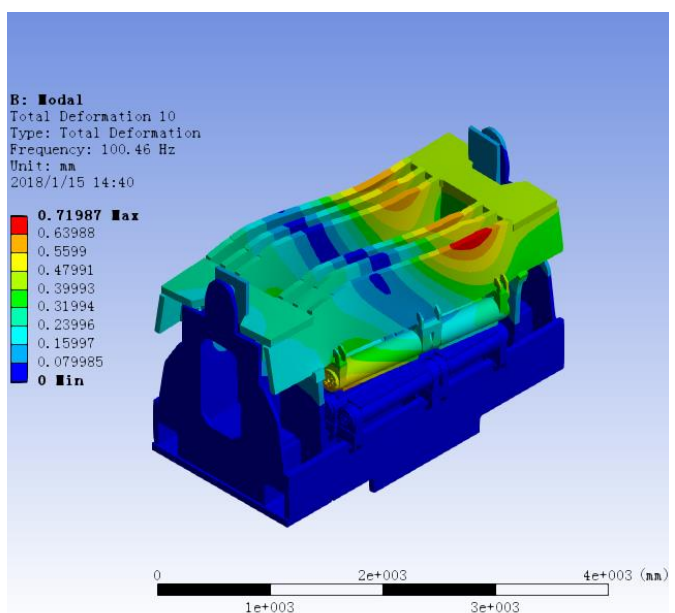

Figure 12. 6th mode shape cloud chart 
The dynamic simulation analysis of the segment frame gives the modal vibration modes and vibration frequencies at various stages of the structure [21]. Observing the first six modes of the segment frame, it can be found that the deformation of the whole structure mainly occurs on the nip of the inner arc frame and the outer arc frame. Among them, the deformations in the first, second, third and sixth mode shapes are mainly occurred in the inner arc frame. The deformations in the fourth and fifth modes are mainly occurred in the nip position of the outer arc frame. The maximum deformation of the inner arc frame occurred in the sixth mode modal, the deformation is $0.71987 \mathrm{~mm}$. The maximum deformation of the outer arc frame nip roll occurred in the fifth mode modal, the deformation is $1.3722 \mathrm{~mm}$.

The deformation of the segment frame is directly related to the force of the model and the application of the constraint. Because there is no direct constraint, the inner arc frame has larger swing and torsion. Because of the fixed constraint, the overall deformation of the outer arc frame is very small. However, due to the pressure load exerted on the nip roller, a greater deformation occurs. The frequencies of the first six modes of the segment frame are mainly distributed in the range of $47.384 \mathrm{~Hz} \sim 100.46 \mathrm{~Hz}$. In order to avoid resonance, the influence of the external excitation of this frequency band on the reliability of the equipment should be avoided.

By analyzing the mode shapes of the frame structures at each natural frequency, it can be seen that the dynamic characteristics of the segment frame are good, and the maximum deformation does not exceed the limit value of $1.8 \mathrm{~mm}$. The results of dynamic analysis of the segment frame provide the basis for the subsequent optimization of the segment frame of the continuous casting machine.

\section{Stiffness test and experimental verification of segment frame}

In order to validate the static and dynamic simulation results, experimental equipment was designed to verify the actual maximum deformation of the segment frame.

\subsection{Design of stiffness test experimental equipment for segment frame}

The hydraulic system has the characteristics of large output force and infinite speed regulation [22]. Therefore, hydraulic jacks were used to simulate the load caused by the static pressure of the slab liquid core on the segment frame, and then the maximum deformation of the frame is measured by the dial indicator. In the experiment, 24 hydraulic jacks were supplied with energy through the hydraulic system. Pressure was applied to the upper and lower driving rollers through the $\mathrm{V}$ block to simulate the load of the slab liquid core and the amount of deformation of the segment frame through the dial indicator. Experimental equipment is shown in Figure 13.

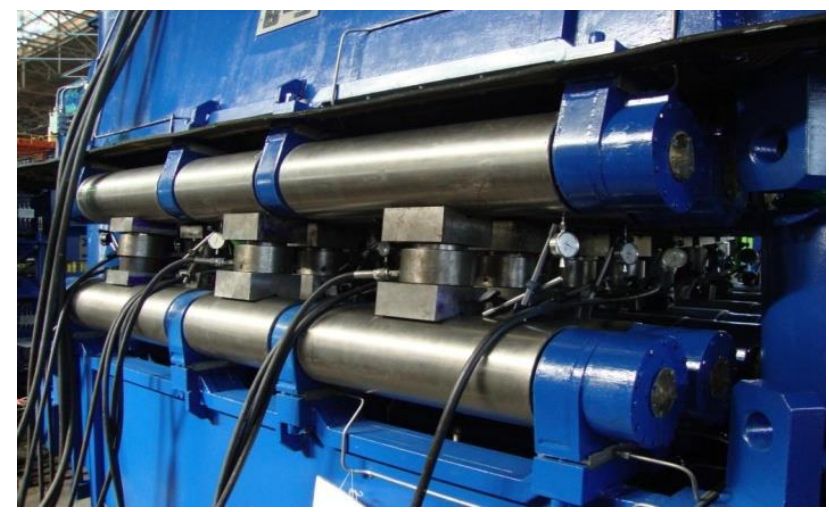

Figure 13. Experimental equipment

Hydraulic jack structure is shown in Figure.14. The upper roller and the lower roller are connected with the frame on the segment to play the role of clamping the slab; the hydraulic jack is arranged 
between the upper roller and the lower roller, and the slab pressure is adjusted and simulated by the expansion and contraction of the piston rod of the hydraulic cylinder; V blocks are placed on both sides of the hydraulic jack, which play the role of connection adapter.

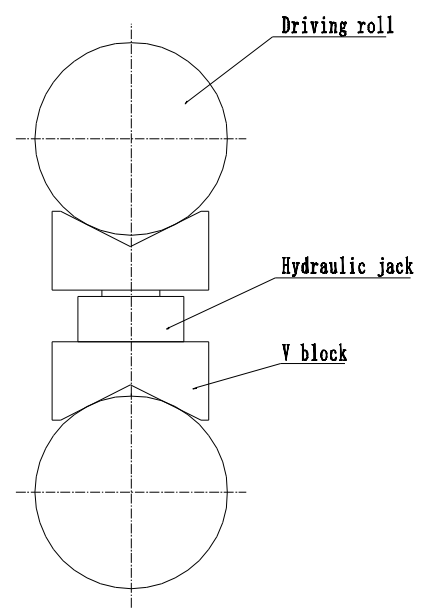

Figure 14. Hydraulic jack structure

\subsection{Discussion}

First, hang the segment section into the test bench, connect the various parts of the hydraulic pipeline; debug clamping cylinder action, exclude hydraulic cylinder gas, add 300mm standard pad, use system pressure $(18 \mathrm{MPa})$ to drive. Put the jacks and pedestals into the segment frame and place a jack (except for the driving roller) on each end of each driving roller, and arrange the jack evenly; install the test dial indicator on the side of each jack and place The number of hands adjusted to " 0 " position; start the experimental hydraulic pump, the pressure from 0 to $1 \mathrm{MPa}$ to $1.5 \mathrm{MPa}$, to eliminate bearing clearance, read the pressure values and record the data; use the $70 \% / 100 \%$ of experimental pressure to withstand the roll surface, so as to ensure good contact between the contact surfaces, record the degree of dial indicator. Finally, remove the test equipment and restore the segment status.

Through many experiments, measure the data of the difference of multiple sets of roll gap through the dial indicator, as shown in Figure15.

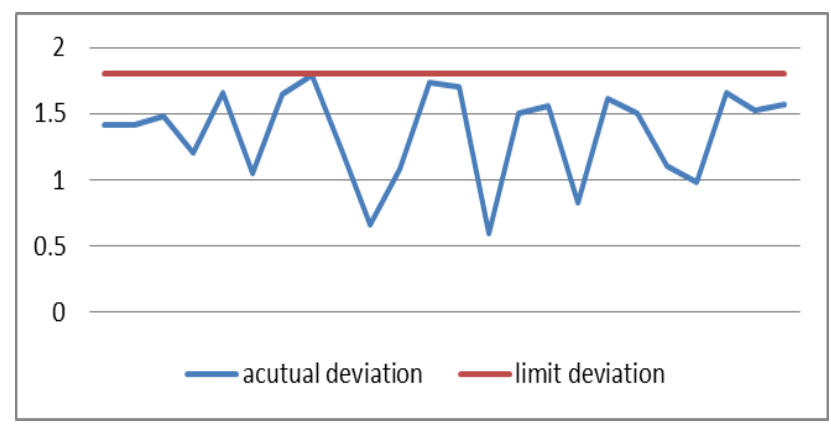

Figure 15. Roll gap actual deviation and limit deviation

The test results show that the maximum deformation of the fan-shaped segment frame occurs at the connection portion of the inner-arc frame pinch roller, where the pinch roller support is weak and the deformation is large. The deformation from the middle of the inner-arc frame to the supporting portion is greatly changed, and the maximum deformation of the roller gap is $1.79 \mathrm{~mm}$, the minimum 
deformation is $0.66 \mathrm{~mm}$, and the results are in good agreement with the simulation and theoretical results.

\subsection{Industrial application}

After the finite element simulation analysis and experimental verification, with dynamic soft reduction, the centerline segregation has been improved, and the maximum deformation of the segment frame can meet the stiffness requirement. Dynamic pressure implementation of effective compensation, the dynamic configuration of the soft reduction of a variety of different steel slab internal quality improvement effect is obvious. Slab internal quality has been significantly improved, slab segregation less than or equal to Class C 1.5 and eliminate the type A sulfur seal. Obviously, with soft reduction, the centerline segregation of steel slab has been improved, as shown in Figure.16.

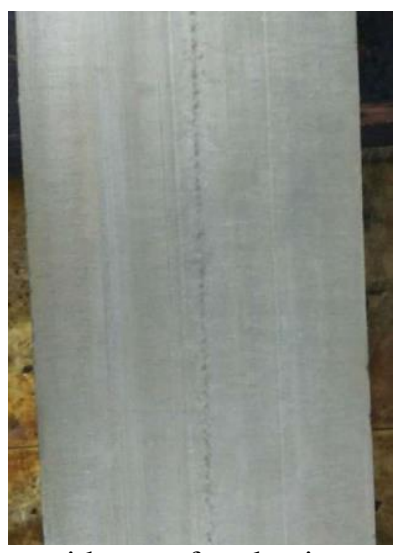

without soft reduction

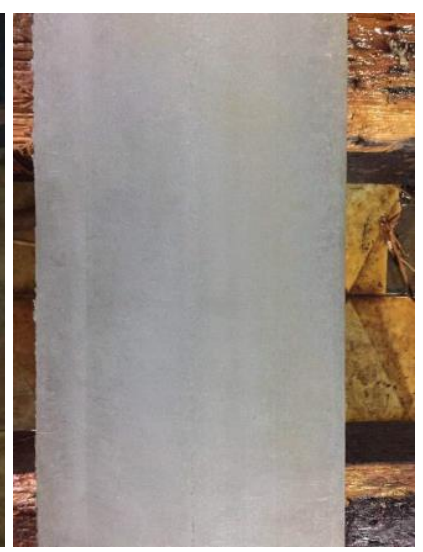

with soft reduction

Figure 16. Application effect

\section{Conclusion}

In order to verify whether soft reduction can improve the stiffness and service life continuous casting machine. In this paper, a three-dimensional model of the segment frame of the continuous casting machine was established. The finite element simulation analysis was carried out to complete the simulation of the maximum deformation and the modal simulation. The simulation results were verified through the hydraulic jack simulation loading test. The experimental results are consistent with the simulation results. The stiffness of the frame structure can meet the requirements with soft reduction.

A three-dimensional solid model of the segment frame was established, and its simplified processing was made to facilitate the finite element simulation. The hexagonal mesh was used to divide the structure so that the calculation accuracy is better and the calculation time is shorter. The maximum deformation cloud, modal vibration frequency and modal vibration modes of the segment frame are obtained through the finite element simulation analysis. On the basis of simulation, the segment frames were loaded through hydraulic jacks, and several experiments were carried out. The experimental results are in good agreement with the simulation results. Segment frame stiffness and dynamic properties are good.

\section{Acknowledgment}

The authors gratefully acknowledge the financial support of Grant-in-aid for scientific research from the National Natural Science Foundation of China (No. 51575429). 


\section{References}

1. K.Ayata, H.Mori, K.Taniguchi, H.Matsuda. Low Superheat Teeming with Electromagnetic Stirring. J, ISIJ Int. 35, 6 ( 1995)

2. M.Javurek, M.Barna, P.Gittler, K.Rockenschaub, M.Lechner. Flow Modelling in Continuous Casting of Round Bloom Strands with Electromagnetic Stirring. J, Steel Res. Int., 79(2008).

3. H.Sun, L.Li, X.Cheng, W.Qiu, Z.Liu, L.Zeng. Reduction in macrosegregation on $380 \mathrm{~mm} \times 490 \mathrm{~mm}$ bloom caster equipped combination M+F-EMS by optimizing casting speed. J, Ironmaking Steelmaking, ,42(2015).

4. J. C. Li, B. F. Wang, Y. L. Ma and J. Z. Cui. Effect of complex electromagnetic stirring on inner quality of high carbon steel bloom. J, Mater. Sci. Eng. A, A425 (2006).

5. V. Ludlow, A. Normanton, A. Anderson, M. Thiele, J. Ciriza, J. Laraudogoitia and W. Knoop. Strategy to minimize central segregation in high carbon steel grades during billet casting. J, Ironmaking Steelmaking, 32 (2005).

6. P. Sivesson, T. Örtlund and B. Widell. Improvement of inner quality in continuously cast billets through thermal soft reduction and use of multivariate analysis of saved process variables. J, Ironmaking Steelmaking, 23(1996).

7. P. Sivesson, G. Häilen and B. Widell: "Improvement of inner quality of continuously cast billets using electromagnetic stirring and thermal soft reduction", Ironmaking Steelmaking, 25(1998).

8. C. M. Raihle, P. Sivesson, M. Tukiainen and H. Fredriksson. Improving inner quality in continuously cast billets: comparison between mould electromagnetic stirring and thermal soft reduction. J, Ironmaking Steelmaking, 21(1994).

9. P. Sivesson, C. M. Raihle and J. Konttinen. Thermal soft reduction in continuously cast slabs. J, Mater. Sci. Eng. A, A173 (1993).

10. W. Bleck, W. Wang and R. Bulte. Influence of soft reduction on internal quality of high carbon steel billets. J, Steel Res. Int., 77(2006).

11. C. H. Yim, J. K. Park, B. D. You and S. M. Yang. The effect of soft reduction on center segregation in c. c. slab. J, ISIJ Int., 36(1996).

12. R. Thome and K. Harste. Study on the design of the soft reduction unit of high-speed billet casters. J, Steel Res. Int., 75(2004).

13. R. Thom and K. Harste. Principles of billet soft-reduction and consequences for continuous casting. J, ISIJ Int., 46(2006).

14. S. Ogibayshi, M. Kobayshi, M. Yamada and T. Mukai. Influence of soft redution with one-piece rolls on center segregation in continuously cast slabs. J, ISIJ Int., 31(1991).

15. J. Cappel, R. Flender, R. Ho“ ffken, G. Kemper and K. Wünnenberg. Centre segregation, soft reduction and oxide cleanness for large diameter line pipe with highest demands on HIC. J, Steel Res. Int., 76(2005).

16. K. Isobe, H. Maede, K. Syukuri, S. Satou, T. Horie, M. Nikaidou and I. Suzuki. Development of soft reduction technology using crown rolls for improvement of centerline segregation of continuously cast bloom. J, Tetsu-to-Hagane, 80(1994).

17. L. Morsut. Technological packages for the effective control of slab casting. J, MPT Int., 25(2003).

18. S. Nabeshima, H. Nakato, T. Fujii, T. Fujimura, K. Kushida and H. Mizota. Control of centerline segregation in continuously cast bloms by continuous forging process. J, ISIJ Int., 35(1995).

19. Gou Wenxuan. Mechanics of Materials (Beijing: Science Press, 2017).

20. Cheng Daxian. Machinery Design Manual Fifth Edition (Beijing: Chemical Industry Press, 2008).

21. Wen DeSheng, Lv Shijun, Wen Jia. New hydraulic system transmission (Beijing: Chemical Industry Press, 2017).

22. Wenjun Wang, Linxin Ning, Raimund Bülte, Wolfgang Bleck. Formation of internal cracks in steel billets during soft reduction. J, Journal of University of Science and Technology, 15 (2008) 